Service social

\title{
La coanimation en travail de groupe : de la théorie à la pratique
}

\section{Isabelle Côté}

Volume 39, numéro 3, 1990

Familles recomposées après divorce

URI : https://id.erudit.org/iderudit/706506ar

DOI : https://doi.org/10.7202/706506ar

Aller au sommaire du numéro

Éditeur(s)

École de service social de l'Université Laval

ISSN

1708-1734 (numérique)

Découvrir la revue

Citer cet article

Côté, I. (1990). La coanimation en travail de groupe : de la théorie à la pratique. Service social, 39(3), 157-169. https://doi.org/10.7202/706506ar
Résumé de l'article

Le document présente quelques bases théoriques de la coanimation en travail de petits groupes. Puis, à partir d'une pratique auprès de nombreux groupes d'enfants, il en établit les avantages et les inconvénients. L'auteure propose que la coanimation est nécessaire dans des problématiques plus difficiles. Elle indique ce qui lui est graduellement apparu être les conditions du succès, de même que les principaux pièges à éviter. Un tableau synthèse résume le document. 


\section{COMMENTAIRES \\ ET DOCUMENTS}

Isabelle Côté, travailleuse sociale au

CLSC Le Phare Sainte-Foy-Sillery.

\section{La coanimation en travail de groupe : de la théorie à la pratique}

Isabelle Côté

Notre pratique au CLSC Sainte-Foy-Sillery nous permet de constater l'utilisation de plus en plus fréquente de la coanimation en intervention de groupe. On la retrouve principalement lorsqu'il s'agit de problématiques complexes (familles recomposées) ou lourdes (violence conjugale). Elle est aussi requise dans l'animation des groupes pour enfants de parents séparés ou divorcés et pour l'expérimentation de nouveaux projets.

De plus, notre recension des écrits sur le sujet, qui s'appuie presque essentiellement sur des données américaines, confirme cet état de choses. Au Québec, des articles récents sur des expériences de groupe dans les CSS et les CLSC signalent et même critiquent l'expérimentation de la coanimation.

Comme ce sujet éveille beaucoup notre intérêt à la fois comme intervenante de groupe en CLSC et comme superviseure, nous avons pensé scruter certains aspects de cette pratique. Le présent article vise à dégager les origines de la coanimation, son évolution, les avantages et les inconvénients qui en découlent. Nous abordons aussi les conditions propices à sa réalisation ainsi que les écueils auxquels les 
intervenants risquent de devoir faire face. En dernier lieu, nous évoquons les applications qui peuvent être faites dans notre pratique ainsi que l'avenir auquel peut prétendre la coanimation.

\section{Les aspects théoriques de la coanimation}

\section{Ses origines}

Heap (1987), reconnaissant la progression notoire de la coanimation tant pour la formation des étudiants que dans les cadres réguliers du travail de groupe, fait ressortir un aspect plutôt paradoxal de cette pratique : son développement rapide malgré l'absence de fondements théoriques et d'évaluation approfondie. Il suggère comme pistes théoriques..." que le travail social de groupe et les domaines voisins de la psychothérapie de groupe et de la thérapie familiale se soient mutuellement influencés..." (p. 180). Une brève incursion chez des spécialistes en thérapie familiale et conjugale (Gullered et Harlan) (1962), Framo (1965), Bellville (1969) tend à confirmer les allégations de Heap. Prenons par exemple ce que ces auteurs donnent comme avantages à utiliser la co-intervention : plus grande sécurité et soutien mutuel pour les co-intervenants, un modèle de référence plus grand pour les clients, l'importance de deux intervenants de sexe différent comme représentation symbolique du père et de la mère, la diminution du temps consacré aux conférences de cas, etc. Ajoutons que la coanimation mixte a aussi comme avantage de fournir aux participants une représentation des rapports hommes-femmes basé, nous l'espérons, sur un partage égalitaire du pouvoir. Ces quelques éléments s'apparentent aux avantages que nous développerons au sujet de la coanimation. Nul doute que nous pourrions aussi trouver des analogies semblables en ce qui a trait aux désavantages ou aux conditions optimales de pratique.

\section{L'évolution dans la formation pratique}

Même sans assises théoriques probantes, la coanimation a pris son envol de façon tellement dynamique que vers la fin des années 70 , les écoles de service social américaines et canadiennes commandaient une recherche sur le sujet. Le but était de circonscrire l'évolution de l'emploi de la coanimation au cours des stages pratiques des étudiants. Cette recherche (questionnaires par la poste) a été conduite auprès de 25 responsables d'écoles universitaires offrant I'approche de groupe. La revue Social Work with Groups (1980) en a donné les résultats au moment de la parution d'un numéro exclu- 
sivement consacré à la coanimation. Beulah Rothman (1980) soulignait que $63 \%$ des écoles rapportaient l'emploi principal de l'animationsolo pour les stages, tandis que $27 \%$ indiquaient que la coanimation était privilégiée. Dans le pourcentage des écoles où l'animation unique avait été choisie en priorité, on relevait cependant que la moitié d'entre elles comptait entre $20 \%$ et $50 \%$ de leurs étudiants qui avaient aussi expérimenté la coanimation dans leur endroit de stage. Les données recueillies faisaient état aussi que de façon très majoritaire les expériences de coanimation se pratiquaient dans les stages en milieux psychiatriques. Elles se faisaient avec un membre d'une autre profession que le service social, soit un psychologue, une infirmière, un psychiatre. L'étude faisait aussi ressortir «the need for social group work educators to conceptualize the desirability of either or both approaches in the education of social workers for group practice $»(\mathrm{p} .14)$.

Intéressons-nous maintenant globalement aux avantages que son utilisation procure.

\section{Les avantages}

Pour étayer le thème des avantages, nous nous référons à Toseland (1984) qui s'inspire des travaux de plusieurs chercheurs (McLennon, 1965; McBee et Sheeman, 1970; Davis et Lorh, 1971; Yalom, 1975; Cooper, 1976; Levine 1980; Starak, 1981; Schelenoff et Busa, 1981). Toseland énumère les avantages qui sont le plus souvent rapportés. Selon ses recherches, la coanimation :

- procure une source de soutien aux animateurs;

- leur permet d'obtenir rapidement une rétroaction de leur animation et favorise ainsi le développement professionnel;

- donne une excellente source de formation à un animateur inexpérimenté;

— procure aux membres du groupe des modèles appropriés de communication, $\mathrm{d}^{\prime}$ interaction et de résolution de conflits;

- permet une complémentarité sur le plan des interventions, particulièrement dans les jeux de rôle, les simulations et autres activités d'animation;

- aide à structurer l'expérience de groupe. (Ex. : Deux coanimateurs ayant des formations professionnelles distinctes peuvent enrichir un groupe dont la problématique traitée nécessite de multiples connaissances bio-psychosociales.)

En plus de tous ces éléments positifs, certains auteurs octroient même à la coanimation un caractère quasi essentiel lorsque se présentent certaines situations difficiles. Nous faisons ici référence à Lan- 
cup et Bélanger (1986) qui citent l'exemple d'une ou deux adolescentes qui arrivent en crise au cours d'une séance de groupe et aussi Larouche (1987) qui décrit le soutien à apporter à une femme en pleine désorganisation durant une rencontre pour femmes violentées. Dans ces situations spéciales, les participantes concernées ont besoin de l'intervention plus individualisée de l'une des animatrices. L'autre peut, pendant ce temps, continuer l'intervention de groupe.

\section{Les désavantages}

Shulmann (1979) retient, à ce sujet, les éléments suivants :

- désaccord sur des principes théoriques de base en intervention de groupe quand il n'y a pas d'ententes préalables;

- lutte de pouvoir pour prendre le contrôle du groupe;

- désaccord pendant le déroulement du groupe en ce qui a trait à des interventions particulières (ex. : court-circuiter la séquence d'intervention d'un coanimateur et l'empêcher ainsi d'aller jusqu'au bout de son idée).

Toseland (1984) mentionne pour sa part que la coanimation est exigeante en matière de temps et d'énergie (rencontres préparatoires, rencontres d'évaluation). II précise aussi que la communication entre les coanimateurs peut devenir difficile si aucun effort concerté n'est fait pour ménager un horaire qui leur permette de discuter de leur travail dans le groupe.

Waldman (1980), quant à elle, pose tout le problème de la relation dominé-dominant qui peut s'établir en coanimation, surtout dans le rapport étudiant-stagiaire vs superviseur. Cela se produit quand les rôles de chacun ne sont pas clairement définis et équilibrés et que l'étudiant se voit contraint ou se complaît dans une fonction plutôt passive. Pour contrer ces difficultés, elle suggère quelques solutions possibles. Le groupe peut être animé par un étudiant qui a une supervision individuelle ou, encore, par deux étudiants avec un statut égal et des rôles communs de coanimateurs avec en plus une supervision partagée. Shulmann (1979), à l'opposé, n'est pas favorable à la coanimation d'un groupe par deux débutants. Il évoque que leur anxiété risque d'être tellement grande qu'ils peuvent se nuire au lieu de s'aider mutuellement. Somme toute, avantages ou désavantages vont souvent de pair avec les conditions que l'on se donne ou non pour faciliter l'exercice de tout projet de coanimation. 


\section{Les conditions inhérentes à la coanimation}

De façon très ferme, Heap (1987 : 182) précise " qu'aucune décision de coanimation ne peut être prise qui ne se fonde sur la primauté des besoins et des sentiments des membres et sur le respect du processus de groupe ». Avant de décider si une coanimation s'avérera nécessaire, il suggère de se poser les questions suivantes (p. 183) :

- «st-ce que cette coanimation facilitera le processus de groupe ou, au contraire, est-ce qu'elle le gênera ?

- Est-ce que les antécédents des membres sont tels que la présence de deux animateurs, ou plus, risque d'augmenter leurs sentiments de malaise et d'infériorité ?"

II va même jusqu'à suggérer un ratio d'un animateur pour trois membres comme un maximum absolu.

Une fois déterminée la primauté de la coanimation, comment choisir la personne qui partagera la fonction de coanimateur ? Doiton opter pour un collègue ayant un même statut et une même expérience comme le suggère Yalom (1975) ? Ou doit-on viser la complémentarité plutôt que la similarité, comme le prétend Heap (1985) ?

Chose certaine, les étudiants et les professionnels qui veulent coanimer doivent être en mesure de partager leurs connaissances théoriques et pratiques, leurs motivations ainsi que leurs attentes bien avant le début du groupe. II est aussi essentiel que dès cette étape, les futurs coanimateurs clarifient les rôles qu'ils entendent jouer, à savoir ceux qui devront être une fonction commune et les autres qui feront appel à leurs habiletés particulières. Ils doivent aussi structurer une grille-horaire prévoyant un échange régulier entre chacune des rencontres du groupe. Un travailleur social du CLSC SainteFoy-Sillery ayant vécu une toute première expérience de coanimation reliée à la problématique de la maladie d'Alzheimer précise que : "bien plus que le nombre élevé de participants (15) et leur hétérogénéité, l'absence d'un temps précis d'échange et de réflexion entre les coanimateurs a pu restreindre l'atteinte de certains objectifs d'aide mutuelle ». Dans le même sens, nous pourrions citer une autre expérience, cette fois-ci auprès de parents d'adolescents et d'adolescentes. Ce nouveau programme était animé par les deux travailleuses sociales affectées aux deux écoles secondaires du territoire desservi par le CLSC. Là encore, une planification faible au chapitre du temps pour préparer le matériel et échanger sur l'animation en cours s'est révélée la pierre d'achoppement du projet bien qu'elle n'en ait pas compromis la réussite.

À la lumière de toutes ces considérations, force nous est de convenir que si l'intervention de groupe ne s'improvise pas, il en est de même pour la coanimation. Shulmann (1979) précise que les mem- 
bres du groupe sont très observateurs et qu'ils peuvent repérer les plus subtils signaux de tension entre les animateurs même si ceux-ci font tout pour les cacher. Offrir un modèle de résolution de conflit aux participants d'un groupe est très aidant. À l'inverse, voir deux animateurs s'empêtrer dans leurs problèmes relationnels peut s'avérer destructeur pour le processus de groupe.

Pour corroborer cette allégation de Shulmann, nous pouvons faire part d'une situation que nous avons personnellement dû affronter. Nous participions avec d'autres intervenants à une formation pratique de plusieurs jours où les deux personnes-ressources, pourtant très expérimentées en groupe, n'ont pu harmoniser leurs compétences particulières et faire alliance pour nous offrir un modèle positif de coanimation. Après un démarrage plutôt réussi, les animateurs en sont venus graduellement à étaler leurs désaccords théoriques, créant ainsi un climat de tension entre eux et avec le groupe. Les participants se sont rapidement divisés en deux factions qui, ouvertement ou de façon plus discrète, avouaient leur préférence pour l'apport de l'un ou de l'autre. Par la suite, certains membres ont aussi menacé de quitter le groupe; d'autres suggéraient aux coanimateurs de régler leur différend, exprimant quant à eux qu'ils n'avaient ni le goût ni l'intention de faire un choix... Les coanimateurs, très pris émotionnellement, ont tout de même réussi à tenir compte des interventions du groupe et ont cherché (et nous ont aussi demandé) des solutions pour améliorer leur fonctionnement. La session a pu se terminer, mais le processus de groupe avait été durement altéré par cet antagonisme. L'analyse ultérieure de ce projet nous permet d'isoler quelques facteurs qui compromettaient dès le départ la coanimation. Ce sont, entre autres, des différences quasi viscérales entre les animateurs en présence et le fait que peu de modalités précises avaient été prévues pour préparer la coanimation, évaluer son adéquation et faire les ajustements qui s'avéraient opportuns.

\section{Les pièges}

Les meilleures conditions réunies n'empêchent toutefois pas la possibilité que certains écueils surgissent pendant une expérience de coanimation. Henry (1981) en énumère quelques-uns :

\section{La division et la conquête des coanimateurs par deux factions du groupe}

L'enjeu qui consiste à faire primer leurs allégeances respectives devient le but ultime des participants dans chacun des sous-groupes. 
Ils escamotent ainsi les thèmes qui devaient être initialement abordés.

Les animateurs devront surveiller l'apparition du moindre signe annonciateur de cette tendance (ex. : deux ou trois participants qui demandent régulièrement et exclusivement de l'information à un des animateurs). Ils devront de nouveau préciser clairement au groupe qu'ils ont tous les deux les mêmes buts et que leur force est de travailler ensemble à l'atteinte des objectifs initialement privilégiés par le groupe.

\section{L'absence d'un coanimateur}

Il est suggéré, quand c'est possible de le faire, d'annoncer à la rencontre précédente l'absence prévue afin que les membres ne la vivent pas comme un abandon ou un rejet. Le coanimateur qui doit animer seul la session doit resituer l'absence de son collègue, réitérer l'importance de la collaboration entre animateurs, ne pas participer " au jeu des comparaisons » et maintenir le focus du groupe sur l'horaire préétabli.

\section{L'intérêt du groupe plus fort pour la relation entre les co- animateurs que pour les orientations propres au groupe}

Ce piège peut être interprété comme une sorte de fantaisie, de curiosité envers des personnes qui représentent une forme de " pouvoir ". Les membres traduisent ainsi la piètre estime qu'ils ont d'euxmêmes, le peu d'importance qu'ils accordent à leurs besoins individuels et de groupe. Heap (1987) mentionne qu'une coanimation mixte (homme et femme) pourrait aussi, en cas de relation très étroite, suggérer une sexualité qui troublerait et gênerait le groupe surtout si les deux animateurs manifestent clairement qu'ils ont, en dehors du groupe, une relation privilégiée. Si cet accent mis sur la relation entre les animateurs se manifeste, ces derniers devront s'ingénier à trouver $\mathrm{d}^{\prime}$ autres moyens plus propices au développement du groupe.

\section{L'utilisation d'un coanimateur comme " coussin de sécurité "}

II arrive qu'un intervenant choisisse un collègue comme coanimateur parce qu'il se sent très à l'aise avec lui, qu'il est son ami. II choisit sciemment son collègue comme soutien affectif et amical, alors que son besoin réel pour son développement professionnel et le succès du groupe serait d'avoir un coanimateur expérimenté capable de lui assurer une formation continue. S. Henry précise qu'aucun travailleur social ne devrait accepter ce rôle de " coussin de sécurité » 
s'il masque le besoin principal et peut contrevenir au processus de groupe.

Après avoir cerné différents aspects théoriques associés à la coanimation, attardons-nous maintenant à son expérimentation en contexte québécois.

\section{Les aspects pratiques de la coanimation en contexte québécois}

Au Québec, cette pratique a touché différentes clientèles : les enfants confrontés au divorce (Côté, 1988), les enfants témoins ou victimes de violence (Alarie et Rose, 1989), les adolescents en milieu scolaire (Lazure, 1980), les adolescentes perturbées clientes d'un CSS (Lancup et Bélanger, 1986), les parents d'adolescents (Turcotte, 1990), les familles d'accueil pour enfants à leur première expérience d'accueil (Gagné et Létourneau-Leblond, 1987), I'intervention de groupe et les femmes violentées (Larouche, 1986). Nous aimerions aussi citer l'expérience novatrice des groupes parents-enfants du CSSMM (Allard, Caron, Durivage, Gélinas, Malo, Ouellette, Rose et Simard, 1989) qui dépasse le cadre de la coanimation et expérimente l'animation en rotation. Les auteurs expliquent cette animation de la façon suivante : "À chaque groupe sont assignés trois animateurs, dont deux permanents. Il y a toujours coanimation. Le troisième animateur travaille davantage à l'évaluation des familles et vient remplacer un des deux permanents de façon périodique. II y a donc une rotation minimale et bien planifiée, assurant une circularité de l'information et du processus de groupe » (p. 40).

À cette énumération qui n'est nullement exhaustive nous pourrions ajouter que la coanimation est omniprésente dans les groupes d'enfants abusés sexuellement $^{1}$, les groupes pour hommes violents, les groupes de soutien pour les familles s'occupant de leurs parents âgés, etc. C'est l'indication que nous donnent les écrits consultés et qui rejoint la réalité que nous vivons aussi dans notre milieu de pratique $^{2}$ et que nous exposons dans le point suivant.

\section{Au CLSC Sainte-Foy-Sillery}

Seulement pour les années 1988-1989 et 1989-1990 (juin 1990), le CLSC Sainte-Foy-Sillery a offert plus d'une cinquantaine de groupes à la population qu'il dessert, la majorité étant donnée en coanimation. Nous pouvons citer :

- les groupes de soutien pour les gens vivant avec une personne atteinte de la maladie d'Alzheimer; 
- les groupes d'entraide pour les familles ayant la garde de leurs parents âgés;

- les groupes pour les adultes et les enfants en situation de rupture conjugale;

- les groupes pour les familles recomposées;

- les groupes de motivation pour les adolescents et adolescentes redoublants;

- les groupes pour les femmes victimes de violence.

De l'ensemble des expériences, il ressort les constatations qui suivent :

- La très forte majorité des groupes étaient coanimés (plus de $70 \%)$.

- La coanimation est exercée presque de façon égale par des intervenants de même souche (ex. : deux travailleurs sociaux) ou appartenant à des professions différentes (ex. : un travailleur social avec une infirmière - un travailleur social avec un orthopédagogue, un travailleur social avec une éducatrice spécialisée, etc.).

- Les groupes animés en solo sont d'abord le fait des stagiaires en service social ( $2^{e}$ stage) ou en counselling-orientation. Ces étudiants bénéficient alors d'une supervision étroite de la part de leurs superviseurs.

- La coanimation est utilisée pour répondre aux besoins du groupe, mais aussi dans un but de formation continue. À une seule reprise, l'organisme a engagé une personne-ressource de l'extérieur pour donner de la supervision en groupe et c'était, notamment, pour superviser le processus de coanimation que vivaient deux travailleuses sociales inexpérimentées en travail de groupe. Ce constat fait dire à un gestionnaire de l'établissement " que la coanimation, malgré ses exigences au niveau des conditions techniques, peut s'avérer non seulement rentable professionnellement mais aussi pas si coûteuse en termes de budget ». Elle serait même plutôt économique si, en plus de répondre aux besoins des clientèles, au soutien pour les coanimateurs et à la complémentarité des connaissances, elle assure une formation continue et la gestion de projets pilotes.

Dans le point suivant, nous illustrerons, à partir d'une problématique particulière, l'apport de la coanimation. 


\section{Notre expérience auprès des groupes d'enfants de la rupture conjugale}

Depuis 1985, nous avons coanimé et supervisé la coanimation de plus de 15 groupes d'enfants de 5 à 12 ans dont les parents vivaient une rupture conjugale. Notre expérience auprès de cette clientèle particulière nous permet $d$ 'apporter les précisions suivantes :

- la coanimation est essentielle pour intervenir auprès de ces jeunes enfants;

- elle permet un meilleur ajustement et réajustement en fonction des besoins des jeunes et de la dynamique du groupe;

- elle actualise une complémentarité des connaissances et activités (réactions sur le divorce, improvisation-marionnettes, etc.);

- elle permet de donner une attention particulière à un enfant en besoin sans freiner le groupe (ex. : un enfant très agité ou en pleurs : un des coanimateurs s'assoit près de lui, le touche pour le calmer, le rassurer);

- elle permet d'assurer la discipline en plus de l'animation;

- elle assure une plus grande sécurité à l'extérieur du lieu physique où se tient le groupe (ex. : pendant qu'une des animatrices accompagne un enfant aux toilettes, l'autre peut poursuivre l'animation et la surveillance du groupe);

- elle permet de s'apporter un soutien mutuel — de souffler un peu.

On pourrait ajouter que les enfants adorent avoir deux animatrices (ils souhaiteraient aussi beaucoup la mixité, mais les intervenants masculins sont rares) et que très vite ils stimulent une participation active de notre part. Un coanimateur pourrait peut-être convenir d'un rôle d'observateur dans un groupe d'adultes, mais jamais dans un groupe d'enfants.

\section{Conclusion}

La recension des écrits et notre survol des expériences québécoises dont celle plus détaillée du CLSC Sainte-Foy-Sillery nous permettent de confirmer la pertinence de la coanimation. Elle satisfait un besoin de soutien par rapport à des clientèles lourdes, de formation continue et de réponses complémentaires à des besoins spéciaux. Les intervenants soutiennent que la coanimation constitue un défi important et stimulant qui demande un important travail de planification et de concertation. Dans l'ensemble, ils semblent y trouver plus d'avantages que de désavantages sans toutefois en faire une panacée. 
La coanimation n'exclut pas l'animation en solo, elle permet plutôt de dynamiser un processus de groupe quand les besoins des clients le commandent. Elle représente des coûts évidents, mais aussi des économies importantes sur le plan de la supervision, sans prétendre toutefois remplacer ce soutien pédagogique lorsqu'il s'avère nécessaire.

\section{Tableau synthèse sur la coanimation}

\section{Cadre théorique}

- Absence de fondements théoriques et d'évaluation approfondie.

- S'inspire des principes de co-intervention de la thérapie familiale et de couple ainsi que de la psychothérapie de groupe.

\section{Utilisation}

- De plus en plus répandue aux É.-U., surtout depuis 1970.

- Au Québec surtout depuis 1980. L'avenir s'annonce prometteur pour les années 90 .

- Beaucoup utilisée auprès des clientèles d'enfants, d'adolescents, de femmes victimes de violence, etc.

- Donc, dans des groupes présentant une problématique lourde ou avec une clientèle où il faut assortir animation, discipline, sécurité.

- Pour démarrer des projets pilotes.

- Utilisée aussi pour la formation d'étudiants en service social de groupe ou pour la formation de professionnels habiles dans une autre approche.

- Va se développer davantage.

\section{Avantages}

- Bon soutien pour les intervenants.

- Bon modèle de relation pour les membres.

- Enrichissement théorique pour les coanimateurs et les membres.

- Aide à maîtriser certaines situations difficiles ou de crise.

- Bonne source de formation, d'encadrement.

- Permet une meilleure complémentarité dans les interventions.

- Aide à structurer l'expérience de groupe.

— Prévient l'essoufflement des professionnels.

\section{Désavantages}

- Coût élevé en temps.

— Difficulté à organiser un horaire pour les coanimateurs (ex. : préparation - évaluation). 
- Danger d'une relation subordonnée pour le stagiaire ou le professionnel néophyte si grandes différences dans le statut et l'expérience.

- Lutte de pouvoir pour prendre le contrôle du groupe.

- Danger de désaccord sur des principes théoriques de base en intervention de groupe.

\section{Conditions de réussite}

- Que la coanimation réponde aux besoins du groupe.

- Que la présence de deux animateurs ou plus n'augmente pas un sentiment de malaise ou d'infériorité dans le groupe.

- Pas plus d'un animateur par trois membres.

- Clarifier, avant le début du groupe, les raisons qui motivent chaque coanimateur.

- Faire une bonne analyse des forces et des faiblesses de chacun.

- Clarifier les rôles respectifs et communs.

- Se donner du temps pour préparer soigneusement les rencontres et évaluer le fonctionnement.

- S'adjoindre un superviseur si nécessaire.

- S'efforcer de comprendre ce que l'autre fait et le soutenir (respect).

\section{Pièges}

- Division et conquête des coanimateurs par deux factions du groupe.

- Absence mal gérée d'un coanimateur.

- Intérêt du groupe plus fort pour la relation entre les coanimateurs que pour les orientations propres au groupe.

- Coanimateur utilisé comme " coussin de sécurité ».

\section{Notes}

\footnotetext{
${ }^{1}$ Nous faisons référence ici à notre participation à un atelier animé par M. James Garland, travailleur social spécialiste des groupes d'enfants, à l'occasion du $11^{\mathrm{e}}$ Symposium de groupe tenu à Montréal à l'automne 1989. La majorité des intervenants présents travaillaient au CSSMM auprès d'enfants abusés sexuellement.

${ }^{2}$ L'auteure tient à remercier ses collègues Ginette Beaulieu, Louise Duval, Monique Thibault, Viviane Vachon qui, à tour de rôle, ont exercé leur compétence dans la coanimation des groupes d'enfants. Elle veut aussi exprimer son appréciation relativement à la participation de plusieurs stagiaires de l'École de service social de l'Université Laval.
} 


\section{Références bibliographiques}

Alarie, F. et D. Rose (1989). " Expérience de groupe auprès d'enfants témoins ou victimes de violence ", Intervention, $\mathrm{n}^{\circ} 83$ : 58-63.

AlLARD, F. et al. (1989). "Groupes parents-enfants ", Intervention, nº 82 : 38-43.

BELLVILLE, T. et al. (1969). "Conjoint marriage therapy with a husband and wife team ", American Journal of Orthopsychiatry, vol. 39, $\mathrm{n}^{\circ} 3$ : 473-483.

CÓtÉ, I. (1988). "Intervention de groupe auprès d'enfants de parents divorcés", Service social, vol. 37, nos 1-2: 214-223.

Framo, J. (1965). Co-therapy team relationship. Rationale and techniques of intensive family therapy. New York : Harper and Row.

GAGNÉ, G. et D. LÉTOURnEAU-LeBlond (1987). Formation en groupe avec des familles d'accueil pour enfants à leur première expérience d'accueil. CSSQ.

Gullered, E. et V. HaRLAN (1962). "Four-way interviewing in marital counseling ", Caseworker, vol. 43, $\mathrm{n}^{\circ} 10: 532-537$.

HeAP, K. (1987). La pratique du travail social avec les groupes. Paris : ESF.

HeNRY, S. (1981). Group Skills in Social Work. A Four Dimensional Approach. Itasca, III. : Peacock publ. inc.

LANCUP, S. et M. BÉLANGER (1986). " Une intervention de groupe auprès d'adolescentes clientes d'un centre de services sociaux ", Service social, vol. 35, $\mathrm{n}^{\circ} 3: 323-338$.

LAROUCHE, G. (1986). "L'intervention de groupe et les femmes violentées", Intervention, $\mathrm{n}^{\circ} 75: 29-36$.

LAROUCHE, G. (1987). Agir contre la violence : La co-animation. Montréal : éd. Pleine Lune.

LAZURE, M. (1980). "Service social de groupe en milieu scolaire auprès d'adolescents ", Service social, vol. 29, $\mathrm{n}^{\text {os }} 1-2$ : 97-111.

RothMAN, B. (1980). "Study of patterns of leadership in group work field instruction ", Social work with groups, vol. 3, $\mathrm{n}^{\circ} 4: 11-17$.

SHULmANN, L. (1979). "Co-workers ", dans The skills of helping individuals and groups. Itasca, III. : Peacock Publishers.

Social Work with Groups (1980). Vol. 3, n² 4, numéro spécial sur la coanimation.

TOSELAND, R.W. et R.F. RIVAS (1984). "Co-leadership », An introduction to group work practice.

TURCOTTE, D. (1990). " Aide professionnelle et groupe d'entraide : une démarche combinée pour les parents d'adolescent(e)s ", Intervention, $\mathrm{n}^{\circ} 85$ : 64-72.

WALDMAN, E. (1980). "Co-leadership as a method of training : A student point of view ", Social Work with Groups, vol. 3, n $1: 51-58$.

Yalom, I.D. (1975). Theory and Practice of Group Psychotherapy (2e éd.). New York : Basic Books. 